\title{
Condensation in ideal Fermi gases
}

\author{
Dragoş-Victor Anghel $\ddagger$ \\ University of Oslo, Department of Physics, P.O.Box 1048 Blindern, N-0316 Oslo, \\ Norway
}

\begin{abstract}
I investigate the possibility of condensation in ideal Fermi systems of general single particle density of states. For this I calculate the probability $w_{N_{0}}$ of having exactly $N_{0}$ particles in the condensate and analyze its maxima. The existence of such maxima at macroscopic values of $N_{0}$ indicates a condensate. An interesting situation occurs for example in 1D systems, where $w_{N_{0}}$ may have two maxima. One is at $N_{0}=0$ and another one may exist at finite $N_{0}$ (for temperatures bellow a certain condensation temperature). This suggests the existence of a first order phase transition. The calculation of $w_{N_{0}}$ allows for the exploration of ensemble equivalence of Fermi systems from a new perspective.
\end{abstract}

PACS number: 05.30.-d, 05.30.Fk, 05.30.Ch

Submitted to: J. Phys. A: Math. Gen.

$\ddagger$ E-mail: dragos@fys.uio.no 


\section{Introduction}

Huge progress have been made in the last decade in cooling trapped Fermi or Bose gases down to the Fermi degeneracy temperature or to the onset of the Bose-Einstein condensation, respectively (see for example Refs. [1, 2, 3, 4, and citations therein). Tuning experimental parameters, like the trap frequency or the magnetic fields applied, one can change the strength of the interaction between the particles and the density of the gas. This freedom, which allows for more accurate comparison between theory and experiment, led to a burst of scientific research in the field. The quantum effects in these gases (fermionic degeneracy and Bose-Einstein condensation) are usually observed at temperatures around or below $1 \mu \mathrm{K}$. Such temperatures can only be achieved by evaporative cooling [5], but the time scale for the cooling process depends strongly on the elastic collision rate of the particles, which determines the thermalization rate [6]. In the case of fermions, at temperatures below the Fermi temperature, the scattering probability falls dramatically due to the Pauli blocking mechanism, which makes cooling very slow. In contrast to this, in the case of bosons, the presence of the Bose-Einstein condensate enhances the cooling rate due to the assisted scattering of hot particles out of the trap, and the cooling process can continue until almost all the gas is condensed. In analogy to the bosonic case, the existence of a condensate in the Fermi system would create the possibility of producing a highly degenerate Fermi gas by removing (evaporating) the particles above the condensate. Moreover, the Fermi condensate might have the same role of enhancing the cooling rate, as the Bose-Einstein condensate. It is well known that a Fermi gas with BCS interaction undergoes a phase transition to a condensed, superconducting phase [7]. In this paper I shall show that a condensate may exist even in ideal Fermi gases.

At low temperatures, there will be a number $N_{0}$ of fermions that occupy the lowest single-particle states. If I number the single particle states from 0 to $\infty$, starting from lowest energy level and going up-wards, then the first unoccupied state is numbered $N_{0}+1$. (The order of the degenerate levels is not important for macroscopic systems.) If $N_{0}$ is a macroscopic number, then I will say that it forms a condensate (see figure 11).

Usually, at low temperatures the particles below the Fermi level are said to be on the "Fermi ground state" (see for example Refs. 8]). The only distinctive physical property of the Fermi energy is that at (very) low temperatures, the particle and hole populations are symmetric with respect to it. Due to the analogy between the BoseEinstein condensate (see Ref. 9]) and the Fermi condensate I suggest that the Fermi condensate may be better suited for the role of Fermi ground state.

In Appendix B of Ref. [10] I showed that a condensate forms in an interacting system (with constant density of states), with general microscopic exclusion statistics properties [11. At the condensation temperature the system undergoes a first order phase transition. In [9] I showed how the condensation in the corresponding noninteracting system changes the character of this phase transition.

\section{Condensation}

Let me consider a Fermi system at temperature $T$ and chemical potential $\mu$. Its grandcanonical partition function is

$$
\mathcal{Z}=\sum_{m} \exp \left[-\beta\left(U_{m}-\mu N_{m}\right)\right],
$$




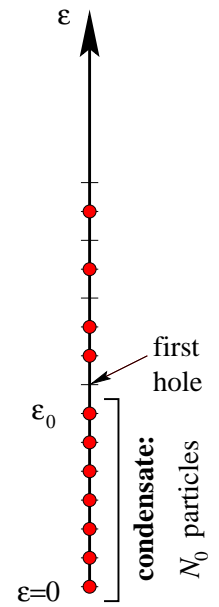

Figure 1. Single particle energy axis. Single-particle states are numbered from 0 to $\infty$, starting from the lowest in energy and going up-wards.

where the sum is taken over all the microstates $m$, of internal energy $U_{m}$ and particle number $N_{m}$; as usual, $\beta \equiv\left(k_{\mathrm{B}} T\right)^{-1}$. If the system is ideal, then $N_{m}=\sum_{i=0}^{\infty} n_{i, m}$ and $U_{m}=\sum_{i=0}^{\infty} n_{i, m} \epsilon_{i}$, where by $\epsilon_{i}$ I denote the energy of the single particle states. I take $\epsilon_{0}=0$ and $\epsilon_{i} \leq \epsilon_{i+1}$, for any $i \geq 0$, as mentioned in the Introduction section. On each single particle state there are $n_{i, m}=0$ or 1 particles. Introducing the expressions for $U_{m}$ and $N_{m}$ into equation (11), I can rewrite $\mathcal{Z}$ in the well-known form,

$$
\mathcal{Z}=\prod_{i=0}^{\infty} \sum_{n_{i}=0}^{1} \exp \left[-\beta\left(\epsilon_{i}-\mu\right) n_{i}\right]=\prod_{i=0}^{\infty}\left\{1+\exp \left[-\beta\left(\epsilon_{i}-\mu\right)\right]\right\}
$$

The logarithm of $\mathcal{Z}$ is related to the grandcanonical thermodynamic potential,

$$
-\beta \Omega \equiv \log \mathcal{Z}=\sum_{i=0}^{\infty} \log \left[1+\mathrm{e}^{\beta\left(\mu-\epsilon_{i}\right)}\right] .
$$

The probability of the microstate $m$ is $w_{m}=\mathcal{Z}^{-1} \exp \left[-\beta\left(U_{m}-\mu N_{m}\right)\right]$.

I assume that for particles in an arbitrary trap and arbitrary number of dimensions, the density of states (DOS) has the expression $\sigma(\epsilon) \equiv C \epsilon^{s}$, where $C$ and $s$ are constants (I take $s>-1$ ). For example if particles of mass $m$ are in a $d$-dimensional box of volume $V$, then $C=V(2 \pi)^{-d / 2} \Gamma^{-1}(d / 2+1) d\left(m / \hbar^{2}\right)^{d / 2}$ and $s=d / 2-1$. I shall use the same procedure as in Ref. [10, to calculate the number of condensed particles. For this, let me calculate the probability that the lowest $N_{0}+1$ states are completely occupied, i.e. $n_{0 \leq i \leq N_{0}}=1$ and $n_{i>N_{0}}$ may be either 0 or 1 . The probability of such a configuration is

$$
\begin{aligned}
\tilde{w}_{N_{0}+1} & =\mathcal{Z}^{-1} \cdot \exp \left[-\beta \cdot \sum_{i=0}^{N_{0}} \epsilon_{i}-\beta \mu\left(N_{0}+1\right)\right] \cdot \prod_{i=N_{0}+1}^{\infty} \sum_{n_{i}=0}^{1} \exp \left[-\beta\left(\epsilon_{i}-\mu\right) n_{i}\right] \\
& =\mathcal{Z}^{-1} \cdot \exp \left[-\beta \cdot \sum_{i=0}^{N_{0}} \epsilon_{i}-\beta \mu\left(N_{0}+1\right)\right] \cdot \prod_{i=N_{0}+1}^{\infty}\left\{1+\exp \left[-\beta\left(\epsilon_{i}-\mu\right)\right]\right\} .
\end{aligned}
$$


Using the expression for $\sigma$ to transform the summation $\sum_{i=0}^{N_{0}} \epsilon_{i}$ into an integral and denoting $\mathcal{Z}_{\text {ex }}\left(N_{0}, \beta, \beta \mu\right) \equiv \prod_{i=N_{0}+1}^{\infty}\left\{1+\exp \left[-\beta\left(\epsilon_{i}-\mu\right)\right]\right\}$, equation (4) becomes

$\tilde{w}_{N_{0}+1}=\exp \left[-\beta C \frac{\epsilon_{0}^{s+2}}{s+2}+\beta \mu C \frac{\epsilon_{0}^{s+1}}{s+1}\right] \cdot \frac{\mathcal{Z}_{\mathrm{ex}}\left(N_{0}, \beta, \beta \mu\right)}{\mathcal{Z}} \equiv \frac{\tilde{\mathcal{Z}}_{N_{0}}}{\mathcal{Z}}$,

where $\epsilon_{0}$ is given by the equation $N_{0}=C \epsilon_{0}^{s+1} /(s+1)$ (is the level up to which all the states are occupied by $N_{0}+1$ particles), while $\mathcal{Z}_{\text {ex }}\left(N_{0}, \beta, \beta \mu\right)$ is the partition function of the particles above the level $\epsilon_{0}$. Note that $\sum_{N_{0}} \tilde{w}_{N_{0}} \neq 1$ and $\tilde{\mathcal{Z}}_{N_{0}=0} \equiv \mathcal{Z}$. $\tilde{w}_{N_{0}+1}$ is the probability that at least $N_{0}+1$ particles are condensed.

The quantity of direct relevance here is the probability to have exactly $N_{0}$ particle condensed. Such a configuration is obtained by removing the particle from the level $\epsilon_{N_{0}}=\epsilon_{0}$. Doing this in equation (44) or (5), I obtain the probability

$w_{N_{0}}=\exp \left[-\beta\left(C \frac{\epsilon_{0}^{s+2}}{s+2}-\epsilon_{0}\right)+\beta \mu\left(C \frac{\epsilon_{0}^{s+1}}{s+1}-1\right)\right] \cdot \frac{\mathcal{Z}_{\mathrm{ex}}\left(N_{0}, \beta, \beta \mu\right)}{\mathcal{Z}} \equiv \frac{\mathcal{Z}_{N_{0}}}{\mathcal{Z}}$.

Note that $w_{\epsilon_{0}} \equiv \sigma\left(\epsilon_{0}\right) w_{N_{0}\left(\epsilon_{0}\right)}$ is the probability density along the energy axis. Nevertheless, in what follows I shall work with $w_{N_{0}}$. If I denote the average of $N_{0}$ by $\tilde{N}_{0} \equiv \sum_{N_{0}} N_{0} w_{N_{0}}$, then $\tilde{N}_{0}$ is macroscopic if (but, in principle, not only if) $w_{N_{0}}$ has a maximum at $N_{0, \text { max }}$, so that in the thermodynamic limit $N_{0, \max } / N>0$, or $\epsilon_{0, \max }>0$ [where $N_{0, \max } \equiv C \epsilon_{0, \max }^{s+1} /(s+1)$ ]. Since $\mathcal{Z}$ does not depend on $N_{0}$, the maximum of $w_{N_{0}}$ may be found by solving the equation $\partial \log \mathcal{Z}_{N_{0}} / \partial N_{0}=0$, or $\partial \log \mathcal{Z}_{N_{0}} / \partial \epsilon_{0}=0$.

First I calculate $\log \mathcal{Z}_{N_{0}}$ from equation (6):

$$
\begin{aligned}
\log \mathcal{Z}_{N_{0}}= & {\left[-\beta\left(C \frac{\epsilon_{0}^{s+2}}{s+2}-\epsilon_{0}\right)+\beta \mu\left(C \frac{\epsilon_{0}^{s+1}}{s+1}-1\right)\right] } \\
& +C \int_{\epsilon_{0}}^{\infty} \mathrm{d} \epsilon \epsilon^{s} \log \left[1+\mathrm{e}^{-\beta(\epsilon-\mu)}\right],
\end{aligned}
$$

where the integral represents $\log \mathcal{Z}_{\text {ex }}\left(N_{0}, \beta, \beta \mu\right)$. The derivative of equation (77) gives

$$
\begin{aligned}
\frac{\partial \log \mathcal{Z}_{N_{0}}}{\partial \epsilon_{0}} & =-C \beta \epsilon_{0}^{s+1}+\beta+C \beta \mu \epsilon_{0}^{s}-C \epsilon_{0}^{s} \log \left[1+\mathrm{e}^{-\beta\left(\epsilon_{0}-\mu\right)}\right] \\
& =-C \epsilon_{0}^{s}\left\{\log \left[1+\mathrm{e}^{\beta\left(\epsilon_{0}-\mu\right)}\right]-\frac{\beta}{C \epsilon_{0}^{s}}\right\},
\end{aligned}
$$

or, without assuming anything about $\sigma, \partial \log \mathcal{Z}_{N_{0}} / \partial \epsilon_{0}=-\sigma\left(\epsilon_{0}\right)\left\{\log \left[1+\mathrm{e}^{\beta\left(\epsilon_{0}-\mu\right)}\right]-\left[\sigma\left(\epsilon_{0}\right) k_{\mathrm{B}} T\right]^{-1}\right\}$.

The interpretation of equation (8) is simple. First note that $\partial \log \mathcal{Z}_{N_{0}} / \partial \epsilon_{0}$ is continuous on the interval $(0, \infty)$. If $\epsilon_{0} \approx \mu$, then $\log \left[1+\mathrm{e}^{\beta\left(\epsilon_{0}-\mu\right)}\right]$ is of the order 1 , so $\partial \log \mathcal{Z}_{N_{0}} / \partial \epsilon_{0}<0$, since in the thermodynamic limit $\left[\sigma\left(\epsilon_{0}\right) k_{\mathrm{B}} T\right]^{-1} \ll 1$. Moreover, for $\beta\left(\epsilon_{0}-\mu\right) \gg 1$,

$$
\frac{\partial \log \mathcal{Z}_{N_{0}}}{\partial \epsilon_{0}} \approx-C \epsilon_{0}^{s} \beta\left\{\epsilon_{0}-\mu-\frac{1}{C \epsilon_{0}^{s}}\right\}
$$

and because $s>-1, \partial \log \mathcal{Z}_{N_{0}} / \partial \epsilon_{0}$ becomes negative for large enough $\epsilon_{0}$, as one would expect (the probability to have an infinite number of particles condensed is zero). To study in more detail the existence and the number of solutions, let me divide the problem into three cases: (1) $s=0,(2) s<0$, and (3) $s>0$. 
Case (1) is the simplest and corresponds, for example, to a Fermi gas in a twodimensional (2D) box. This case may be analyzed in connection with the Bose-Fermi thermodynamic equivalence in (2D), which was outlined in Section 2.2 of Ref. [10. The properties of ideal 2D Bose gases have been studied extensively in the past (see for example [12] and citations therein). For $s=0$ equation (8) becomes:

$$
\left.\frac{\partial \log \mathcal{Z}_{N_{0}}}{\partial \epsilon_{0}}\right|_{s=0}=-C\left\{\log \left[1+\mathrm{e}^{\beta\left(\epsilon_{0}-\mu\right)}\right]-\frac{1}{C k_{\mathrm{B}} T}\right\} .
$$

If I take the thermodynamic limit simply as $\left(C k_{\mathrm{B}} T\right)^{-1}=0$, then $\partial \log \mathcal{Z}_{N_{0}} /\left.\partial \epsilon_{0}\right|_{s=0}<$ 0 for all $\epsilon_{0}$ and $T$, so there is no condensate. On the other hand, note that for $\beta\left(\epsilon_{0}-\mu\right) \ll-1, \log \left[1+\mathrm{e}^{\beta\left(\epsilon_{0}-\mu\right)}\right] \approx \mathrm{e}^{\beta\left(\epsilon_{0}-\mu\right)}$ and, because of the exponential dependence on $\beta\left(\epsilon_{0}-\mu\right)$, the term in curly brackets in equation (9) may become negative even for a macroscopic system. Since $\log \left[1+\mathrm{e}^{\beta\left(\epsilon_{0}-\mu\right)}\right]$ is monotonically increasing with $\epsilon_{0}$, while $\left(C k_{\mathrm{B}} T\right)^{-1}$ is a constant, equation (9) has maximum one solution, and this must be for $0 \leq \epsilon_{0}<\mu$. I define the condensation temperature

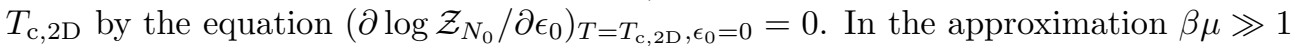
this condition gives:

$$
\frac{\mu}{k_{\mathrm{B}} T_{\mathrm{c}, 2 \mathrm{D}}}=\log \left[\left(k_{\mathrm{B}} T_{\mathrm{c}, 2 \mathrm{D}}\right) C\right] .
$$

For any $T>T_{\mathrm{c}, 2 \mathrm{D}}$, equation (9) has no solution, while for $T \leq T_{\mathrm{c}, 2 \mathrm{D}}$ it has one and only one solution for $\epsilon_{0} \geq 0$, so the system is condensed. To see if equation (10) has any relevance for macroscopic systems, let me consider a 2D gas of electrons of Fermi energy $\epsilon_{\mathrm{F}}^{\mathrm{Al}}=11.7 \mathrm{eV}$ (Fermi energy of electrons in $\mathrm{Al}$ ). If I take the area of the 2D gas to be $S=1 \mathrm{~m}^{2}$ (surely a macroscopic system) and $\mu=\epsilon_{\mathrm{F}}^{\mathrm{Al}}$, then

$$
T_{\mathrm{c}, 2 \mathrm{D}}^{\mathrm{Al}} \approx 3263 \mathrm{~K} \text {. }
$$

At room temperature $T_{\mathrm{r}}=300 \mathrm{~K}$, keeping $\mu=\epsilon_{\mathrm{F}}^{\mathrm{Al}}$, I obtain $\epsilon_{0, \text { max }}^{\mathrm{Al}} \approx 10.7 \mathrm{eV}$, only about $1 \mathrm{eV}$ smaller than $\mu$. If I take $\tilde{N}_{0} \approx N_{0, \max }$, then the condensate fraction $\left(\tilde{N}_{0} / N\right)$ is $\epsilon_{0, \text { max }}^{\mathrm{Al}} / \epsilon_{\mathrm{F}}^{\mathrm{Al}} \approx 0.91$.

The condensation energy scales roughly with the chemical potential. For another example I take heavily doped silicon with the doping concentration of $n=4 \times 10^{25} \mathrm{~m}^{-3}$ 21. For this concentration, the Fermi energy is $\epsilon_{\mathrm{F}}^{\mathrm{Si}} \approx 0.04 \mathrm{eV}$, which gives a condensation temperature of about $14 \mathrm{~K}$. In this case, the condensate fraction at, say $100 \mathrm{mK}$ (roughly the working temperature for the microcoolers or microbolometers), is over $99 \%$.

Two systems of equal number of particles are called thermodynamically equivalent if they have the same entropies as function of temperature. All 2D ideal gases of equal DOS are thermodynamically equivalent, irrespective of their microscopic exclusion statistics 13, 14, 15, 16, 17, 18, 19, 20, 10, and this is due to the similarity between their excitation spectra [10. If I put in correspondence the Fermi system under investigation with the equivalent Bose system, and I denote by $\mu_{\mathrm{B}}$ the chemical potential of the later, then $\mu_{\mathrm{B}}=\mu-\epsilon_{\mathrm{F}}$ (where $\epsilon_{\mathrm{F}}$ is the Fermi energy). The number $N_{0}$ of fermions in the condensate is equal to the number of bosons on the ground state, say $N_{\mathrm{B}, 0}$ [10]. Moreover, in the canonical ensemble the condensate fluctuations are the same for the two, equivalent, Bose and Fermi gases.

Case (2) is maybe the most interesting. For $s<0, \partial \log \mathcal{Z}_{N_{0}} / \partial \epsilon_{0}<0$ in both limits. $\epsilon_{0} \rightarrow 0$ and $\epsilon_{0} \rightarrow \infty$, so eventual solutions of the equation $\partial \log \mathcal{Z}_{N_{0}} / \partial \epsilon_{0}=0$ 
come in pairs. To see that there is only one pair of solution note that that the derivative $\partial \log \left[1+\mathrm{e}^{\beta\left(\epsilon_{0}-\mu\right)}\right] / \partial \epsilon_{0}=\beta /\left[\exp \left(\beta \mu-\beta \epsilon_{0}\right)+1\right]$ increases with $\epsilon_{0}$, so $\log \left[1+\mathrm{e}^{\beta\left(\epsilon_{0}-\mu\right)}\right]$ is a function concave up-wards, while $1 / C \epsilon_{0}^{s}$ is a function concave down-wards $(-1<s<0)$. Therefore the two curves $\left(\log \left[1+\mathrm{e}^{\beta\left(\epsilon_{0}-\mu\right)}\right]\right.$ and $\left.\beta / C \epsilon_{0}^{s}\right)$ may cut each-other either in exactly two points, or in zero points. Moreover, if I assume that they do cut and at $\epsilon_{0} \approx \mu$, or bigger, then $k_{\mathrm{B}} T$. $\left\{\partial \log \left[1+\mathrm{e}^{\beta\left(\epsilon_{0}-\mu\right)}\right] / \partial \epsilon_{0}\right\}=\left[\exp \left(\beta \mu-\beta \epsilon_{0}\right)+1\right]^{-1}$ is of the order one or bigger, while $-k_{\mathrm{B}} T \cdot\left[\mathrm{d}\left(1 / C \epsilon_{0}^{s}\right) / \mathrm{d} \epsilon_{0}\right]=-s k_{\mathrm{B}} T /\left[C \epsilon_{0}^{s} k_{\mathrm{B}} T \cdot\left(\epsilon_{0} / k_{\mathrm{B}} T\right)\right] \ll 1$, in the thermodynamic limit. Therefore, $\partial\left\{\log \left[1+\mathrm{e}^{\beta\left(\epsilon_{0}-\mu\right)}\right]-\frac{\beta}{C \epsilon_{0}^{s}}\right\} / \partial \epsilon_{0}>0$ for $\epsilon_{0} \approx \mu$, or bigger. Since $\log \left[1+\mathrm{e}^{\beta\left(\epsilon_{0}-\mu\right)}\right]-\frac{\beta}{C \epsilon_{0}^{s}}>0$, for $\epsilon_{0} \approx \mu$, I conclude that $\partial \log \mathcal{Z}_{N_{0}} / \partial \epsilon_{0}=0$ has no solutions for $\epsilon_{0} \in[\mu, \infty)$, no matter what is the value, or sign of $\mu$. If $\partial \log \mathcal{Z}_{N_{0}} / \partial \epsilon_{0}=0$ has solutions, then $\mu>0$ and the solutions are in the interval $\epsilon_{0} \in(0, \mu)$.

I define the condensation temperature by the equation

$$
\left.\max _{\epsilon_{0}} \frac{\partial \log \mathcal{Z}_{N_{0}}}{\partial \epsilon_{0}}\right|_{T=T_{\mathrm{c}, \mathrm{s}<0}}=0 .
$$

For $T>T_{\mathrm{c}, \mathrm{s}<0}$, equation (8) has no solution, so the system is not condensed. Particles on a one-dimensional (1D) interval have $\sigma(\epsilon)=C \epsilon^{-1 / 2}$, so I shall take $1 \mathrm{D}$ electron systems as examples. If I take again the Fermi energy of Al for electrons on an interval of length $l=1 \mathrm{~m}$, I obtain $T_{\mathrm{c}, 1 \mathrm{D}} \approx 6242 \mathrm{~K}$.

The interesting aspect about the $s<0$ case is that below the condensation temperature $\mathcal{Z}_{N_{0}}\left(\epsilon_{0}\right)$ has two maxima: one at $\epsilon_{0}=0$ and one at $\epsilon_{0}=\epsilon_{0, \max }>0$. By comparing the areas below the peaks, one can conclude which maximum is the stable solution and which is the metastable one. Similar to the situation outlined in Appendix B of Ref. [10, this leads to a first order phase transition from an uncondensed, to a condensed phase, in 1D canonical Fermi systems.

Case (3) includes three-dimensional (3D) ideal systems, which correspond to $s=$ $1 / 2$. If $s>0$, obviously $\partial \log \mathcal{Z}_{N_{0}} / \partial \epsilon_{0}>0$ at $\epsilon_{0}=0$. Since $\log \left[1+\mathrm{e}^{\beta\left(\epsilon_{0}-\mu\right)}\right]$ is monotonically increasing and $\left(C k_{\mathrm{B}} T \epsilon_{0}^{s}\right)^{-1}$ is monotonically decreasing, while $\partial \log \mathcal{Z}_{N_{0}} / \partial \epsilon_{0}$ is negative at large $\epsilon_{0}$, I conclude that exists one and only one finite $\epsilon_{0, \max }$, and therefore a finite $N_{0, \max }$, at which $w_{N_{0, \max }}$ attains its maximum. For example for one cubic meter of $\mathrm{Al}$, at room temperature, $\epsilon_{0, \max } \approx 11.2 \mathrm{eV}$ and the condensate fraction is about $94 \%$.

An important example is taken from the experiments performed by D. Jin and collaborators [2, 3] and I shall use parameters from Ref. 3]. I describe $N=6 \times 10^{6}$ atoms of ${ }^{40} \mathrm{~K}$ (I take both spin polarizations), confined in a $3 \mathrm{D}$ harmonic trap of (geometric) mean frequency $\omega / 2 \pi=70 \mathrm{~Hz}\left(\hbar \omega \approx 2.9 \times 10^{-11} \mathrm{eV}\right)$. The DOS is $\sigma(\epsilon)=\epsilon^{2} /(\hbar \omega)^{3}$ and the Fermi energy is $\epsilon_{\mathrm{F}}=(3 N)^{1 / 3} \hbar \omega \approx 7.6 \times 10^{-11} \mathrm{eV}$. With this parameters I calculate two values of $\epsilon_{0, \max }: \epsilon_{0, \max }\left(T_{1}=1 \mu \mathrm{K}\right) \approx 2.6 \times 10^{-12} \mathrm{eV}$ and $\epsilon_{0, \max }\left(T_{2}=300 \mathrm{nK}\right) \approx 1.3 \times 10^{-11} \mathrm{eV}$. Note that both, $\epsilon_{0, \max }\left(T_{1}\right)$ and $\epsilon_{0, \max }\left(T_{2}\right)$ are smaller than $\hbar \omega$, so there is no condensate, although the equation $\partial \log \mathcal{Z}_{N_{0}} / \partial \epsilon_{0}=0$ has a solution. Eventually the possibility to obtain a highly degenerate Fermi gas is to agglomerate may particles in a trap and then to separate the condensate formed at the "bottom" of the trapping potential. 


\section{Conclusions}

In this paper I discussed the formation of a condensate in ideal Fermi systems with the density of single particle states of the form $\sigma(\epsilon)=C \epsilon^{s}$ (where $C$ and $s$ are constants). I did this by calculating the probability $w_{N_{0}}$ of having exactly $N_{0}$ particles in the condensate. If $s>0$ (like in 3D boxes or traps), $w_{N_{0}}$ has a maximum for $N_{0}>0$, which indicates the formation of a condensate. The maximum persists at any temperature, but eventually $N_{0}$ becomes microscopical as $T$ increases. For a cubic meter of $\mathrm{Al}$ at room temperature, $N_{0} / N \approx 0.94$ (where $N$ is the total number of particles in the system). Unfortunately for the current experiments in harmonic traps, $N_{0}$ appears to be smaller than one, which means that there is no condensate. Maybe a high increase of particle number in the trap, without much concern for the temperature, would lead to the formation of a condensate, which may be afterwords separated by evaporating the uncondensed particles.

The case $s=0$ corresponds to 2D boxes or 1D harmonic traps. This is equivalent to a 2D Bose gas and the results of canonical calculations may be interchanged down to the microscopic scale, with proper redefinition of single particle energies (see Ref. [10]). After the interchange, the particles in the Fermi condensate become the particles onto the Bose ground state, which have been extensively studied [12].

Maybe the most interesting case is $s<0$. Here $w_{N_{0}}$ may have two maxima. One maximum is always at $N_{0}=0$ (no condensate), while the other maximum forms at finite $N_{0}$, for temperatures below a condensation temperature. The existence of the two maxima suggest a phase transition of order one in canonical Fermi systems and a reconsideration of the ensemble equivalence. In this category are included 1D Fermi systems.

\section{References}

[1] A. J. Leggett, Rev. Mod. Phys. 73, 307 (2001); K. M. O'Hara, S. L. Hemmer, M. E. Gehm, S. R. Granade, and J. E. Thomas, Science 298, 2179-2182 (2002); A. G. Truscott, K. E. Strecker, W. I. McAlexander, G. B. Partridge, and R. G. Hulet, Science 291, 2570 (2001).

[2] B. DeMarco and D. S. Jin, Science 285, 1703 (1999).

[3] B. DeMarco, S. B. Papp, and D. S. Jin, Phys. Rev. Lett. 86, 5409 (2001).

[4] F. Dalfovo, S. Giorgini, L. P. Pitaevskii, and S. Stringari, Rev. Mod. Phys. 71, 463 (1999).

[5] H. F. Hess, Phys. Rev. B 34, 3476 (1986).

[6] M. J. Holland, B. DeMarco, and D. S. Jin, Phys. Rev. A 61, 053610 (2000).

[7] J. Bardeen, L. N. Cooper, and J. R. Schrieffer, Phys. Rev. 108, 1175-1204 (1957); N. N. Bogoliubov, Nuovo Cimento 7, 794 (1958); Soviet Phys.-JETP 7, 41 (1958).

[8] M. N. Tran, M. V. N. Murthy, and R. K. Bhaduri, Phys. Rev. E 63, 031105 (2001); M. N. Tran, J. Phys. A: Math. Gen. 36961 (2003).

[9] D. V. Anghel, cond-mat/0310377

[10] D. V. Anghel, J. Phys. A: Math. Gen. 35, 7255 (2002); cond-mat/0105089

[11] F. D. M. Haldane, Phys. Rev. Lett 67, 937 (1991).

[12] M. Holthaus, E. Kalinowski and K. Kirsten, Ann. Phys. (N.Y.) 270, 198 (1998); M. Holthaus and E. Kalinowski, Ann. Phys. (N.Y.) 276, 321 (1999).

[13] R. M. May, Phys. Rev. 135, A1515 (1964).

[14] M. H. Lee, J. Math. Phys. 36, 1217 (1995).

[15] M. H. Lee, Phys. Rev. E 56, 3909 (1997).

[16] M. H. Lee, Phys. Rev. E 55, 1518 (1997).

[17] M. H. Lee and J. Kim, Physica A 304, 421 (2002).

[18] M. Apostol, Phys. Rev. E 56, 4854 (1997).

[19] S. Viefers, F. Ravndal, and T. Haugset, Am. J. Phys. 63, 369 (1995).

[20] S. B. Isakov, D. P. Arovas, J. Myrheim, A. P. Polychronakos, Phys. Lett. A 212, 299 (1996); D. Sen and R. K. Bhaduri, Phys. Rev. Lett. 74, 3912 (1995). 
[21] A. M. Savin, M. Prunnila, P. P. Kivinen, J. P. Pekola, J. Ahopelto, and A. J. Manninen, Appl. Phys. Lett. 79, 1471 (2001). 